\title{
GRAIN BOUNDARY MIGRATION AND THE TEXTURE OF FILMS
}

\author{
IDAJEAN M. FISHER and DAVID A. SMITH
}

IBM T. J. Watson Research Center, Yorktown Heights, NY 10598, USA

\begin{abstract}
Extensive studies of the microstructure of deposited films establish that grain boundary migration is a ubiquitous process in the development of microstructure and frequently the key process in the formation of a preferred orientation. This conclusion is supported by the interpretation of observations of the structure and orientation of films as a function of substrate temperature and post deposition annealing. Epitaxial deposits can result from oriented nucleation or selective growth processes. On amorphous substrates the anisotropy of the interfacial energies of the deposit results in a fiber texture at temperatures when grain growth occurs either during deposition or in a post-deposition anneal.
\end{abstract}

KEY WORDS Grain boundary migration, nucleation, fiber texture, bimodal distribution.

\section{INTRODUCTION}

The microstructure of a deposited film represents the outcome of a number of processes: adsorption, surface diffusion, nucleation clustering, coarsening and grain growth. The kinetics of each of these processes and their respective contributions to the microstructure of the film are particularly sensitive to the nature and temperature of the substrate and the cleanliness of the system. Thermodynamic calculations of the critical nucleus size and observations, reviewed by Venables and Price (1975), both support the view that the grain structure of a film is invariably only distantly related to the nucleation process or processes. The grain structure is however, directly related to the islandcoalescence and grain boundary migration processes that occur during deposition; the rates of these processes are both governed by thermally activated transport. This is the scientific basis to the observation that the substrate temperature, (expressed in terms of the melting point of the deposit in $\mathrm{K}$ ), is a general guide to the microstructure of films irrespective of the material being deposited, the deposition process or the nature of the substrate (Thornton, 1975; Grovenor, Hentzell and Smith, 1984). With this in mind it is appropriate briefly to review the essential kinetics of grain boundary migration as a prelude to the discussion of experimental data.

\section{GRAIN BOUNDARY MIGRATION}

Movement of grain boundaries is a thermally activated process which involves the transfer of an atom or possibly a group of atoms from one grain to a neighbor across a grain boundary. The kinetics of grain boundary migration can be 
analyzed in terms of absolute rate theory. On a one dimensional argument the velocity, $v$, of the interface is given by absolute rate theory as follows (e.g. Christian 1965)

$$
v=r A \mu\left(\frac{\Delta \mu}{k T}\right) \exp \left(-\frac{\Delta g}{k T}\right)
$$

where $r$ is the distance moved per activated event, $A$ is a geometric factor, $\Delta \mu$ the chemical potential change driving the process, $\Delta g$ the free energy of activation, usually comparable with that for grain boundary diffusion, and $k T$ has its usual meaning; $r, A, \mu$ and $\Delta g$ are all in principle structure dependent. In addition it is likely in theory (Cahn, 1962), and verified experimentally that the activation energy in Eq. 1 can be increased by solute pinning and decreased by a supersaturation of vacancies (Aust and Rutter, 1959; Atwater, Thompson and Smith, 1987); consequently since $\Delta g$ can be approximately halved or doubled, respectively, the grain boundary mobility is profoundly affected by excess vacancies or impurities.

\section{OBSERVATIONS}

Figure 1 is a bright field transmission electron micrograph and the corresponding diffraction pattern of a molybdenum film deposited at $648 \mathrm{~K}\left(0.22 T_{m}\right)$ onto a

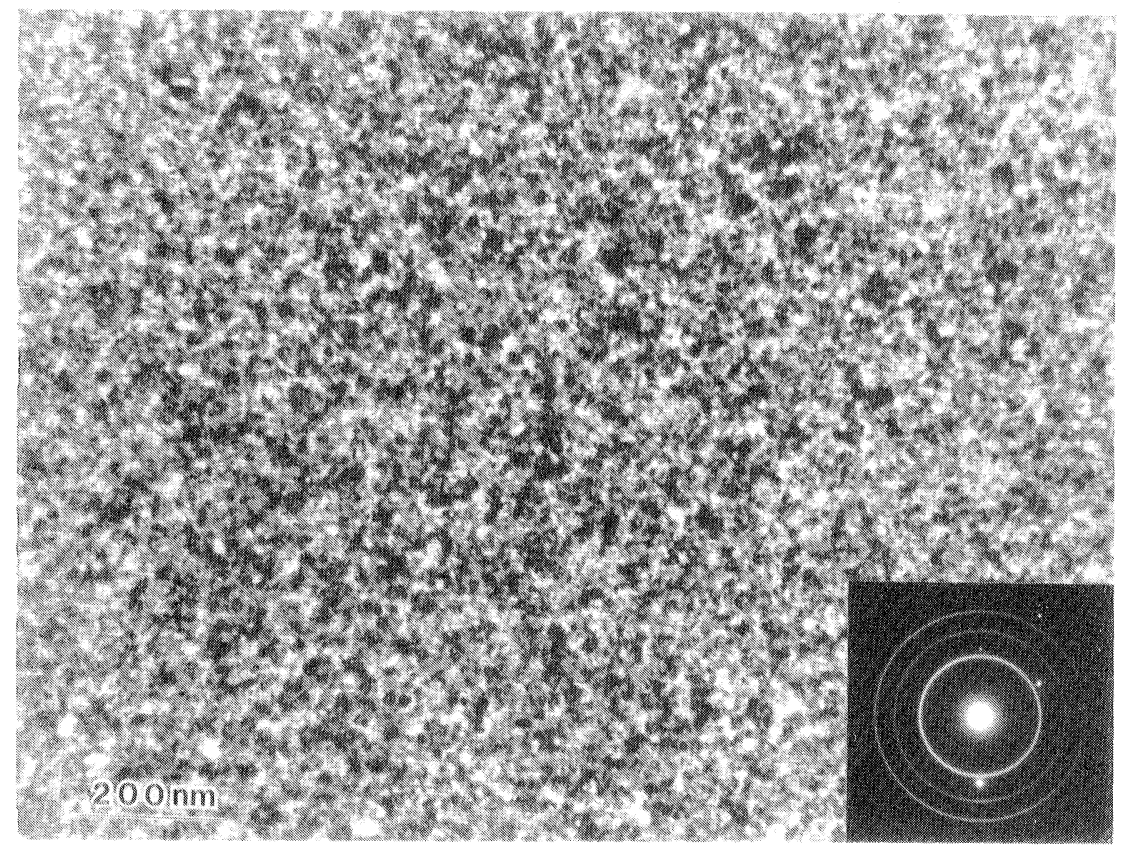

Figure 1 A bright field transmission electron micrograph and the corresponding diffraction pattern of a molybdenum film deposited at $648 \mathrm{~K}\left(0.22 T_{m}\right)$ onto a [111] oriented wafer of GaAs. 
$\{111\}$ oriented wafer of GaAs. The $20 \mathrm{~nm}$ grain size and the random orientation relationship revealed by the diffraction pattern are characteristic of metallic films deposited in diffusion pumped systems with the substrate at or below about $0.2 T_{m}$. The substrate exerts no effect on the orientation of the deposit. The remarkable aspect of the deposit shown in Figure 1 is that it is crystalline rather than amorphous. There is evidently sufficient atomic mobility to permit the growth of crystalline grains but the relatively low substrate temperature and high, rather than ultra-high, vacuum precludes sufficient mobility for the development of a textured deposit by selective grain boundary migration. This microstructure is associated with transient grain boundary mobility. In a UHV environment, deposition at the same substrate temperature results in epitaxial growth (Eizenberg et al., 1986). Relative to the behavior of bulk materials it is a surprising observation that grain growth should occur at all at a homologous temperature of $0.22 T_{m}$.

However when tantalum is deposited, even at a slightly lower temperature, $573 \mathrm{~K}$, onto gold, in a diffusion pumped system, a strongly oriented deposit results, this is illustrated in Figure 2, which shows aligned islands of tantalum on a doubly positioned $\{111\}$ oriented gold thin film substrate. This is an example of oriented nucleation. The orientation relationship between the deposit and the substrate can be understood in terms of minimization of misfit between the substrate and the deposit which serves to direct the nucleation process by giving the low energy nuclei an overwhelming free energy advantage. Evidently the resistance of the gold substrate to contamination is sufficient to ensure behavior which is related to the interaction of the substrate and the deposit rather than to that of adventitious impurities. This result is even more remarkable, in comparison to the behavior of molybdenum on GaAs, since the melting point of tantalum is $3273 \mathrm{~K}$.

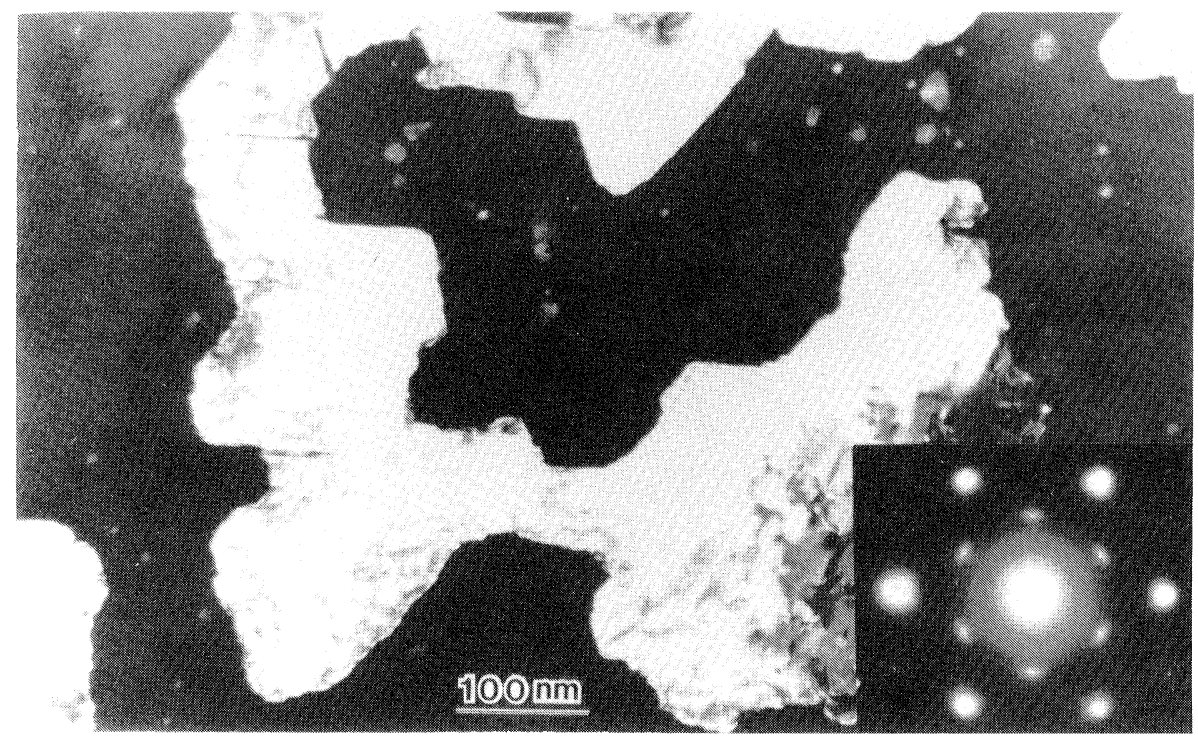

Figure 2 A dark field transmission electron micrograph which shows aligned islands of tantalum on a doubly positioned [111] oriented gold thin film substrate. 
Even when the substrate is amorphous the crystallographic variation of the surface energy of the deposit can result in preferred orientation (Winterbottom, 1967; Taylor and Cahn, 1988). The anisotropies of the substrate-deposit and the deposit-ambient interfacial energies must each be considered. A consequence of growth under conditions where interfacial energies are minimized can be a fiber texture. For continuous films the mechanism is the preferential growth of grains with one or more low energy surfaces, (Usually a single grain extends through the film). The preferential growth of a grain with low surface energy is driven by a force of the order $\left(\Delta \gamma+\Delta \gamma_{s d}\right) / h$ where $h$ is the film thickness and the subscript $s d$ signifies the substrate-deposit interface; note that the magnitude of this surface energy difference will be greater by a factor of perhaps three for a free surface relative to that of the substrate-deposit interface. Thus under conditions of marginal mobility the greater driving force at the free surface would result in more grain boundary motion at that surface consistent with the tapered grains (narrow end nearer to the substrate) which are often observed in cross-sectional samples.

The role of surface energy in biassing the growth of certain grains is illustrated in Figure 3 which is a transmission electron micrograph of a gold film $e$-beam deposited at room temperature onto amorphous silicon nitride; the film is capped with a thin $\mathrm{SiO}_{2}$ layer to minimise pinning of grain boundaries by surface grooving during post deposition anneals (Mullins, 1958). The film thickness is $30 \mathrm{~nm}$ and the large grains are shown to share a $\langle 111\rangle$ normal and to be arbitrarily oriented azimuthally, as shown by the convergent beam diffraction pattern of Figure 3a. The $\{111\}$ fiber texture is evidenced by the anomalously intense 220 diffraction ring of Figure $3 b$.

The $\{111\}$ fiber texture sharpens and the grain size distribution evolves to larger dimensions, but remains bimodal, as a consequence of deposition at a higher temperature or annealing at temperature higher than the deposition temperature. These phenomena are illustrated in Figures $4 a, b$ and $c$ for gold, as deposited at $294 \mathrm{~K}$, and then heated to $873 \mathrm{~K}$ and for gold deposited at $473 \mathrm{~K}$. The substrate is amorphous silicon nitride. The insets in each figure are the corresponding selected area diffraction patterns. Figures $4 \mathrm{~d}, \mathrm{e}$ and $\mathrm{f}$ are histograms representing the distribution of grain sizes corresponding to the structures shown in Figures $4 a, b$ and $c$ respectively.

As a last example of the effect of grain boundary migration on texture evolution the origin of the "epitaxial temperature" is considered (Khan 1970). This concept is an expression of the fact that it is necessary to exceed a critical temperature in order to assure the deposition of a single crystal film in weakly interacting systems such as the noble metal-alkali halide deposit-substrate couples. In contrast to the behavior of molybdenum on gold, Figure 2 which is an example of a system where there is evidently a strong azimuthal dependence of the interfacial energy, the noble metals on alkali halides, exemplified by gold on salt, nucleate in a wide range of orientations and the "epitaxial" configuration is established during the island growth and coalescence processes. The morphologies of films at the liquid-like coalescence stage below and above the "epitaxial temperature" are very similar but the internal structures differ significantly. The critical difference is that in the former case stable triple grain junctions have formed and these are absent in the latter case. This is illustrated in Figures $5 \mathrm{a}$ and $\mathrm{b}$. 


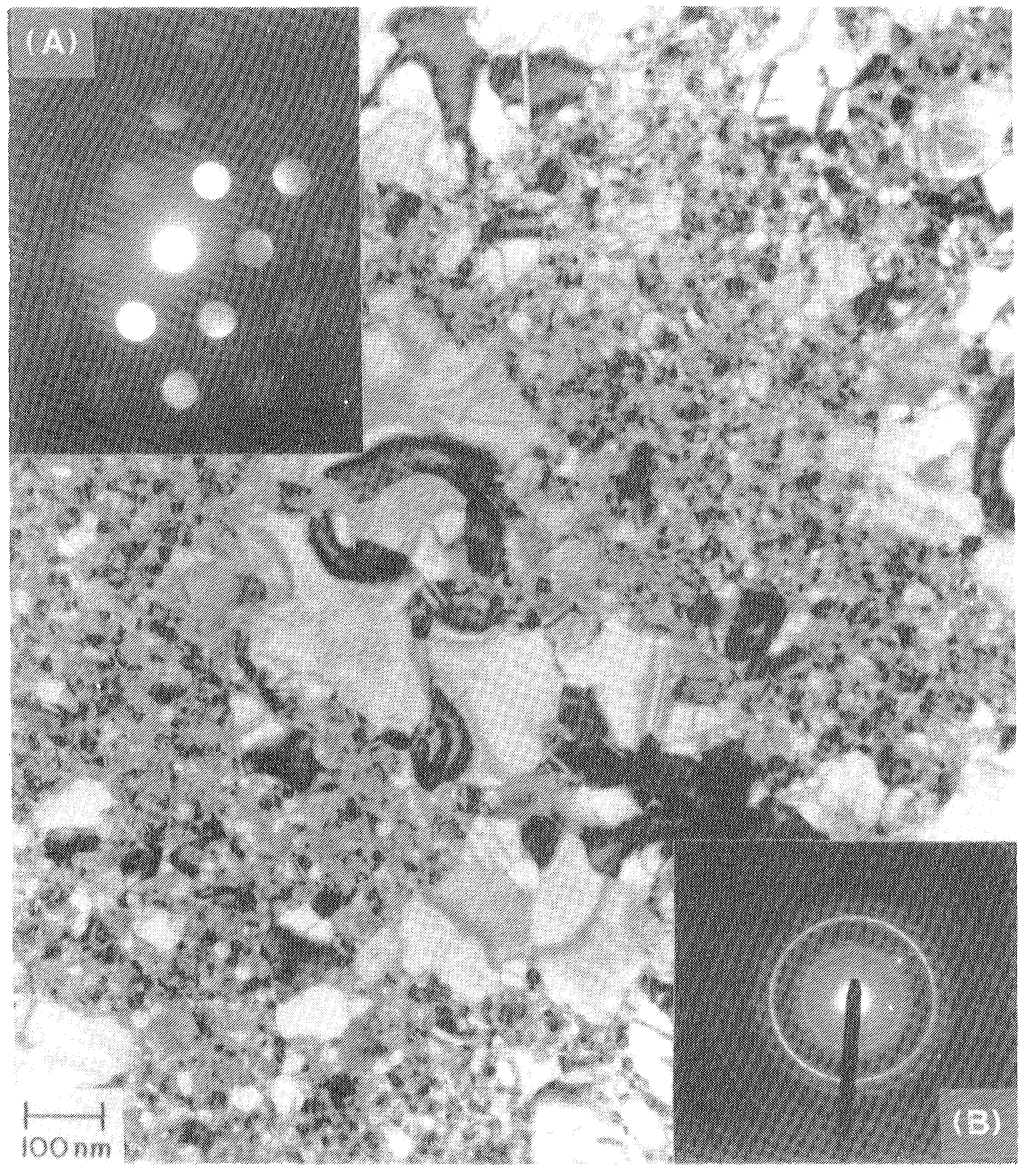

Figure 3 A transmission electron micrograph of a gold film deposited at room temperature onto amorphous silicon nitride. The grain size distribution is clearly bimodal. The anomalously intense 220 diffraction ring of inset (B) along with the convergent beam pattern of inset (A) are evidence for a $\{111\}$ fiber texture. The convergent beam pattern is representative of the orientation of the large grains.

\section{DISCUSSION}

The application of classical nucleation theory to condensation from the vapor leads immediately to the prediction that the critical nucleus has a radius of the same order as that of an atom. This result of course implies that the calculation, which is based on macroscopic thermodynamic quantities is not to be construed quantitatively. The qualitative conclusion that the grain sizes observed in films are large even compared with the radius of a homogeneous formed nucleus formed by condensation from the vapor is valid. Experimental data from, for example, field-ion microscopy (Smith, 1989) and reflection high energy diffraction (Eizenberg et al., 1986) together suggest that condensation and crystallisation of the 


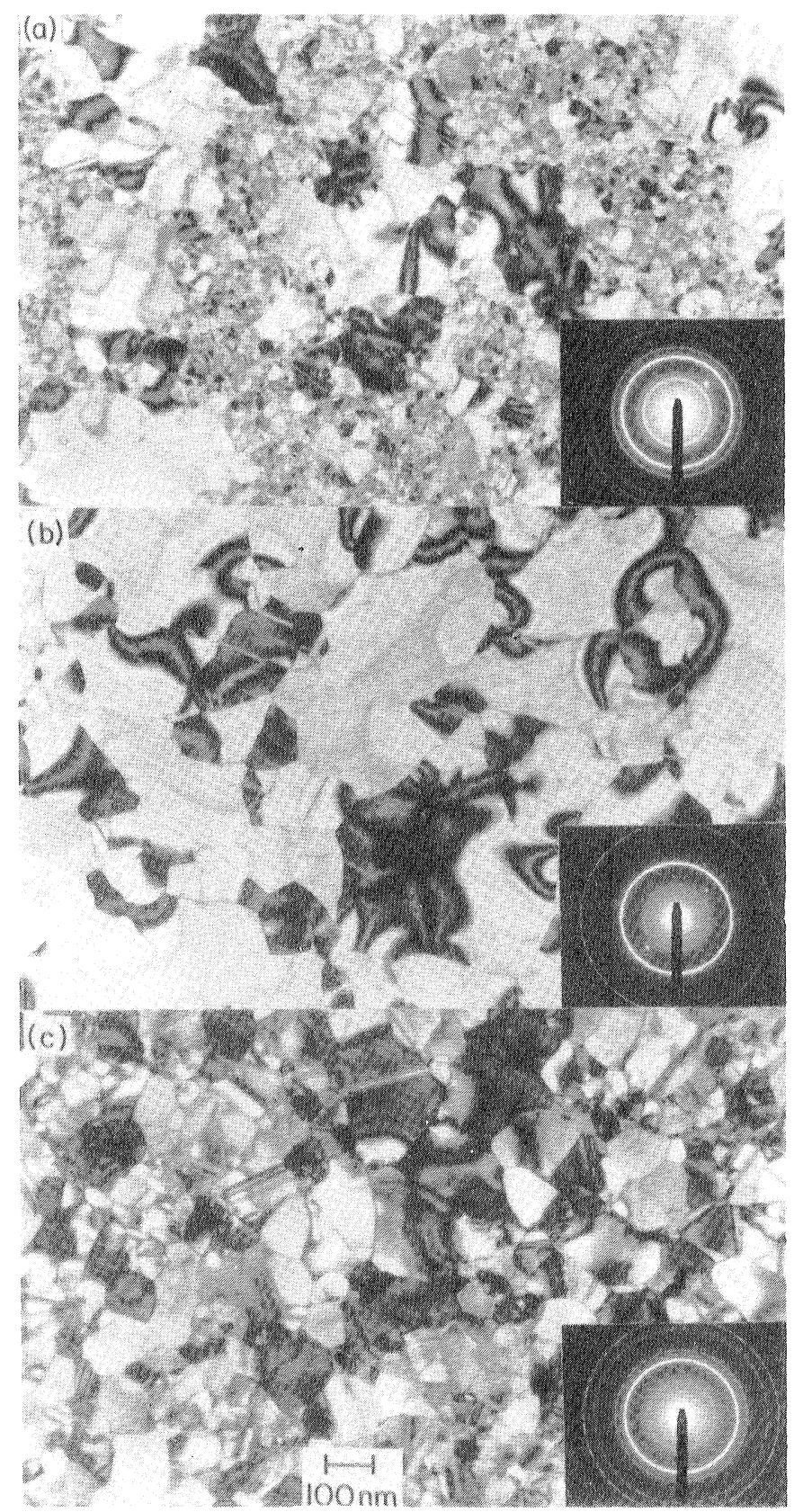

Figures 4 Bright field transmission electron micrographs of yold (a) as deposited at $294 \mathrm{~K}$, (b) deposited at $294 \mathrm{~K}$ and then heated to $873 \mathrm{~K}$, and (c) as deposited at $473 \mathrm{~K}$. The substrate is amorphous silicon nitride. The insets in each figure are the corresponding selected area diffraction patterns. 

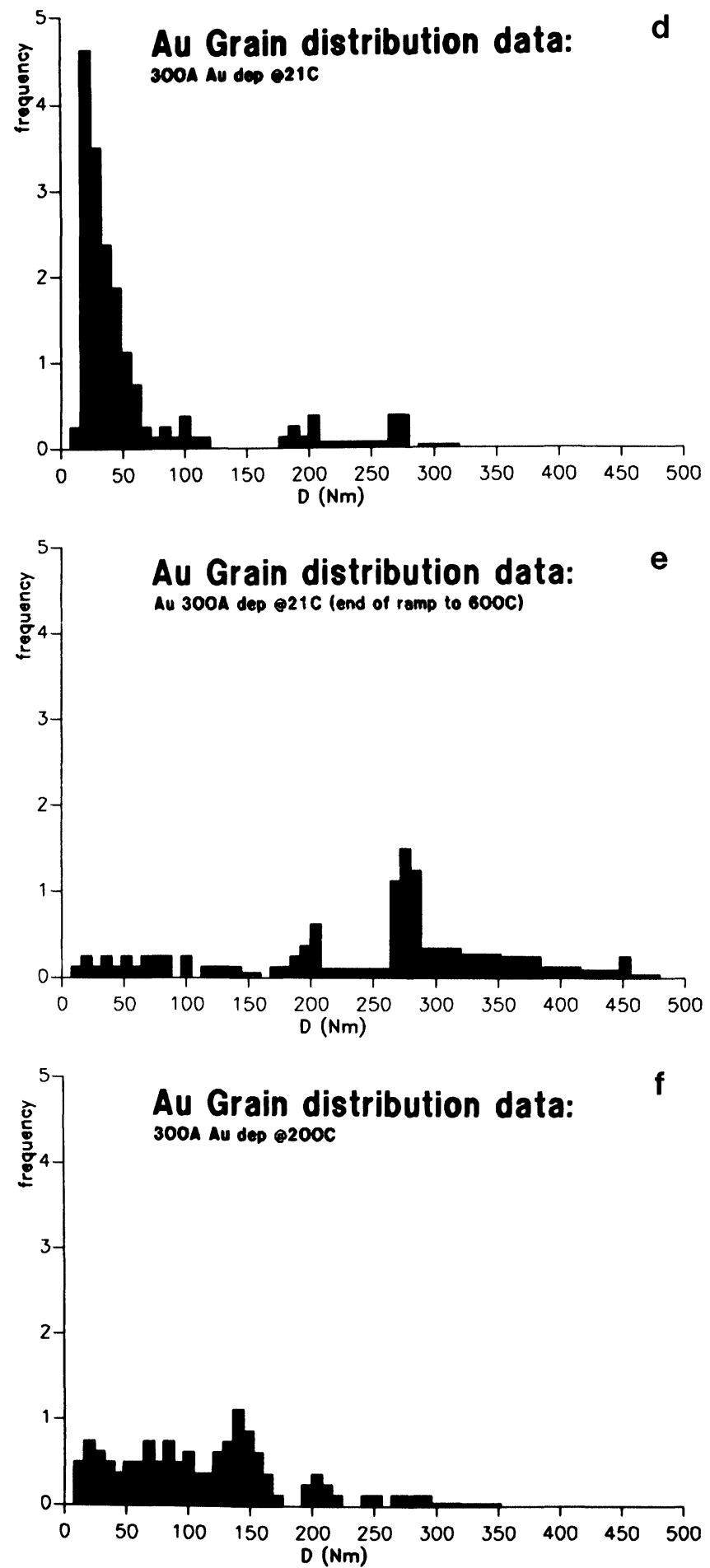

Figure 4 (Continued) (d), (e) and (f) are histograms representing the distribution of grain sizes corresponding to the structures shown in (a), (b) and (c) respectively. 

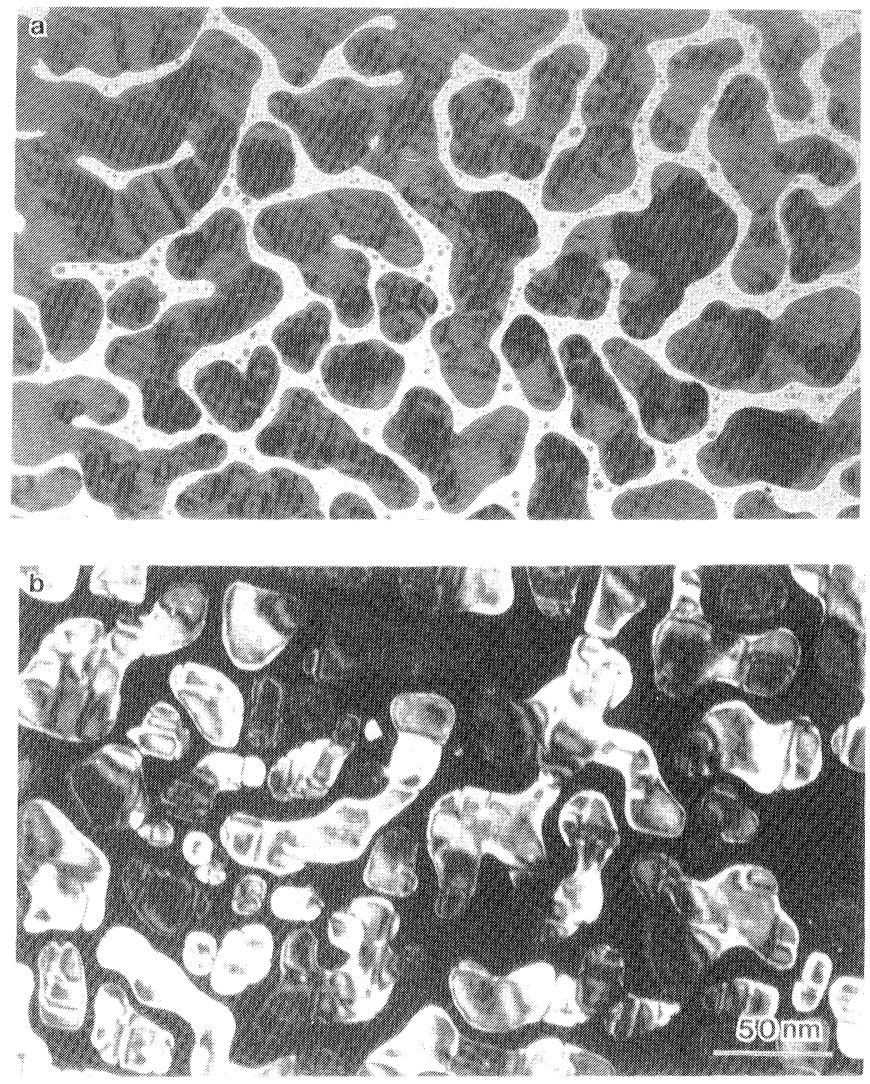

Figure 5 The morphologies of gold films at the liquid-like coalescence stage below and above the "epitaxial temperature"; the critical difference is that in (a) stable triple grain junctions have formed and these are absent in (b).

equilibrium solid phase are separate processes and thus the calculation of a radius for a critical nucleus for the condensation process may be misleading. However grain growth evidently exerts an overwhelming effect on the final microstructures reported in this paper since the grain morphology and grain size distributions are inconsistent with nucleation followed by growth to impingement (Tu, Smith, and Weiss, 1987).

In principle the texture of a film is a result of the combined effects of surface energy anisotropy on the nucleation and grain growth processes. The orientation of the molybdenum islands on gold is consistent with minimisation of interfacial energy at the nucleation stage. However for the gold where extensive grain growth occurs during deposition no texture effects attributable to nucleation were observed; the $\{111\}$ texture was established by the selective growth of those grains bounded by low energy external surfaces.

Grain boundaries are not thermodynamically stable in metals but are almost invariably present because of the formation of metastable configurations of which the triple junction is the prototype. The key to the formation of single crystal 
deposits in island growth systems is to adjust the growth conditions, most easily the substrate temperature, to eliminate the formation of triple junctions. Raising the substrate temperature or decreasing the deposition rate both favor grain boundary elimination relative to island coalescence.

As a final point of discussion it is worth commenting on the unusually low temperatures at which grain growth occurs in deposited films. This behavior is entirely consistent with the unusually large capillary driving force and the likely supersaturation of point defects in a growing film.

\section{CONCLUSION}

Bias of nucleation and grain growth processes to minimise anisotropic surface and interfacial energies accounts for the textures observed in as-deposited or post-deposition annealed films.

\section{References}

1. Atwater, H. A., Thompson, C. V. and Smith, H. I. (1988). Ion-bombardment-enhanced grain growth in germanium, silicon and gold thin films. Journal of Applied Physics, 64, 2337-2353.

2. Aust, K. T. and Rutter, J. W. (1959). Temperature Dependence of Grain Boundary Migration in High-Purity Lead Containing Small Additions of Tin. Transactions of the Metallurgical Society of AIME, 215, 820-831.

3. Cahn, J. W. (1962). The Impurity Drag Effect in Grain Boundary Motion. Acta Metallurgica, 10, 789-798.

4. Christian, J. W. (1965). Theory of Transformations in Metals and Alloys, pp. 87-94. Oxford: Pergamon Press.

5. Eizenberg, M., Smith, D. A., Heiblum, M. and Segmuller, A. P. (1986). Electron Beam Evaporation of Oriented Nb Films onto GaAs Crystals in UHV. Applied Physics Letters, 49, 422-424.

6. Grovenor, C. R. M., Hentzell, H. T. G. and Smith, D. A. (1984). The Development of Grain Structure during Growth of Metallic Films. Acta Metallurgica., 32, 773-781.

7. Khan, I. H. (1970). The Growth and Structure of Single-crystal Films. In Handbook of Thin Film Technology, edited by L. I. Maissel and R. Glang, pp. 10-1 to 10-65. New York: McGraw-Hill.

8. Mullins, W. W. (1958). The Effect of Thermal Grooving on Grain Boundary Motion. Acta Metallurgica, 6, 414-427.

9. Smith, D. A. (1989). Interfaces and Thin Film Microstructure. In Proceedings of EMAG-MICRO 89:, edited by P. J. Goodhew, Bristol: Institute of Physics.

10. Taylor, J. E. and Cahn, J. W. (1988). Theory of Orientation textures due to Surface Energy Anisotropies. In Microstructural Science for Thin Film Metallisations in Electronics Applications, edited by J. Sanchez, D. A. Smith and N. DeLanerolle, pp. 73-77. Warrendale: The Minerals, Metals and Materials Society.

11. Thornton, J. A., (1975). Influence of Substrate Temperature and Deposition Rate on the Structure of Thick Sputtered Copper Coatings. Journal of Vacuum Science and Technology, 12, 830-835.

12. Tu, K. N., Smith, D. A. and Weiss, B. Z. (1987). Hyperbolic Grain Boundaries. The Physical Review B, 36, 8948-8950.

13. Venables, J. A. and Price, G. L. (1975). Nucleation of thin films. In Epitaxial Growth, edited by J. W. Matthews, pp. 381-436. New York: Academic Press.

14. Winterbottom, W. L. (1967). Equilibrium Shape of a Small Particle in Contact with a Foreign Substrate. Acta Metallurgica, 15, 303-310. 